\title{
A Simple Fask Protocol as an Effective Screening Tool for Patients With Abdominal Distention
}

\section{Wan-Ching Lien}

Department of Emergency Medicine, National Taiwan University Hospital, Taipei, Taiwan https://orcid.org/0000-0001-9448-5093

\section{Jia-Yu Chen}

Department of Emergency Medicine, National Taiwan University Hospital, Taipei, Taiwan

\section{Pei-Hsiu Wang}

Department of Emergency Medicine, National Taiwan University Hospital, Taipei, Taiwan

\section{Dean-An Ling}

Department of Emergency Medicine, National Taiwan University Hospital, Taipei, Taiwan An-Fu Lee

Department of Emergency Medicine, National Taiwan University Hospital, Taipei, Taiwan

\section{Ying-Chih Ko}

Department of Emergency Medicine, National Taiwan University Hospital, Taipei, Taiwan

\section{Yu-Tse Hsiao}

Department of Emergency Medicine, National Taiwan University Hospital, Taipei, Taiwan

\section{Chien-Kai Wu}

Department of Emergency Medicine, National Taiwan University Hospital, Taipei, Taiwan

\section{Wei-Tien Chang}

Department of Emergency Medicine, National Taiwan University Hospital, Taipei, Taiwan

\section{Chien-Hua Huang}

Department of Emergency Medicine, National Taiwan University Hospital, Taipei, Taiwan

\section{Hsiu-Po Wang ( $\square$ wanghp@ntu.edu.tw )}

Department of Internal Medicine, National Taiwan University Hospital, Taipei, Taiwan d Department of Internal Medicine, College of Medicine, National Taiwan University, Taipei, Taiwan

\section{Original research}

Keywords: point-of-care ultrasound, abdominal distention, ascites, distended urinary bladder, discharge, length of stay

Posted Date: August 10th, 2020

DOI: https://doi.org/10.21203/rs.3.rs-52530/v1 
License: (c) (i) This work is licensed under a Creative Commons Attribution 4.0 International License. Read Full License 


\section{Abstract}

Background: The retrospective study aimed to investigate the effect of point-of-care ultrasound (PoCUS) by a designated protocol for patients with abdominal distention.

Methods: Non-traumatic adult patients with abdominal distention were included at the emergency department (ED) of the National Taiwan University Hospital between July 2015 and July 2017. A sonographic scanning protocol (FASK, the focused assessment with sonography in trauma plus the renal US) was included in the post-graduate year and residency US training. The primary outcome included the diagnostic accuracy of the FASK protocol. The accuracy, sensitivity, specificity, positive predictive value (PPV), and negative predictive value (NPV) of the protocol were calculated that discharge/admission diagnosis was as the standard. The secondary outcomes included ED LOS and the rate of ED discharge using the FASK protocol.

Results: A total of 512 patients were included. The accuracy of the FASK protocol was $99.0 \%(507 / 512)$, whatever the level of the performer. The sensitivity, specificity, PPV, and NPV were $99.4 \%$ (95\% Cls, $98.2-$ 99.9\%), 100\% (95\% Cls, 100\%), 99.6\% (95\% Cls, 98.6-100\%), and 99\% (95\% Cls, 97.7-99.7\%), respectively. 397 patients (78\%) were discharged with the median LOS of 126 minutes (IQR, 84-236 minutes) after receiving PoCUS and proper management. PoCUS was performed approximately 3 hours earlier than CT (median, 179 minutes; IQR, 90-468 minutes; $p<0.0001$ ).

Conclusions: A simple FASK protocol could be an effective screening tool for non-critical patients with abdominal distention. Being an adjunct of physical examination and management, PoCUS exhibited characteristics of efficacy, timeliness, and safety.

\section{Trial registration: NCT04149041 at ClinicalTrials.gov.}

\section{Background}

Abdominal distention is a common complaint in emergency care settings. Diverse mechanisms participate in the pathogenesis of abdominal distension. Therefore, it becomes a challenge to diagnose accurately, and manage properly [1]. Traditionally, thorough history taking and physical examination are essential for evaluation for patients with abdominal distention. However, limitations exist because of the chaotic environment, the decline of bedside physical examination, and the increase in ambiguity in diagnosis [2,3].

Ultrasound (US) is a safe and rapidly evolving diagnostic modality [3]. Currently, reducing the size of modern devices and improving image quality make point-of-care US (PoCUS) ideal for immediate bedside use [4], and suitable for patient care. It can serve as a valuable adjunct to physical examination [5] and facilitate patient care and disease management, referring to "the stethoscope of the 21 st century" [6, 7]. The use of PoCUS first can answer important clinical questions accurately without time delay, and avoid side effects of unnecessary, advanced imaging [8]. PoCUS has shown its value in rapidly diagnose critical 
conditions such as shock, dyspnea, and cardiac arrest [9-12]. Evidence regarding the application of PoCUS in non-critical patients with abdominal distention was limited in the literature [13, 14]. Also, whether PoCUS could enhance the efficiency of the management process and influence patient-oriented outcomes such as length of stay (LOS), and the rate of discharge was still uncertain [15].

We conducted a retrospective study to investigate the effect of PoCUS by a designated protocol for patients with abdominal distention.

\section{Methods}

\section{Study design and setting}

The retrospective study was conducted at the emergency department (ED) of the National Taiwan University Hospital, a tertiary medical center in Taiwan, between July 2015 and July 2017. The ED has an annual uptake of approximately 85,000 patients. The study protocol was approved by the institutional review board of the hospital (201907173RIND) with a waiver of informed consent and registered at ClinicalTrials.gov (NCT04149041).

The sonographic scanning protocol (FASK) for abdominal distention included the focused assessment with sonography in trauma (FAST) examination detecting free fluid over Morison's pouch, splenorenal recess, and within the pelvis, plus urinary tract examination including bilateral kidneys and urinary bladder. The distended urinary bladder was identified as an estimated urinary bladder volume more than $400 \mathrm{ml}[16,17]$. This protocol was included in the post-graduate year (PGY) and residency US training since 2012, and all of the PGYs and residents passed the hand-on assessment under the senior instructors' supervision. The instructors were certified by the Society of Ultrasound in Medicine, Republic of China, Taiwan, and had over 10 years of experience in sonographic examinations.

All ultrasonographic examinations were written in a standard report form including indication, sonographic findings, sonographic diagnosis, and management. A US machine (SSA-550A, Canon, Japan) equipped with 2-5 MHz curvilinear transducers was kept ready for use.

\section{Selection Of Participants}

Adult non-traumatic, ED patients more than 20 years of age (according to the Regulations on Human Trials conducted since 2016 in Taiwan) with abdominal distention receiving PoCUS with the FASK protocol were included. Patients aged less than 20 years, with pregnancy or trauma, and receiving PoCUS for other indications were excluded. Critical patients with cardiac arrests and shock were also excluded.

\section{Measurements}


Clinical data were obtained, including age, gender, body mass index (BMI), vital signs at arrival (blood pressure, heart rate, pulse saturation, and body temperature), registration time, door to US time, door to computed tomography (CT) time, ED LOS, whether ED discharge or not. The accuracy of sonographic diagnosis was defined as the agreement between sonographic diagnosis and discharge/admission diagnosis (presence of ascites, urinary bladder distention, or none of both).

All sonographic images and documentation were collected, as well as the level of the sonographer. The images were reviewed by two instructors blindly and independently. The quality of the images was categorized using a 5-point Likert rating scale. Point 1 indicated no recognizable structures, no objective data can be gathered; point 2 indicated minimally recognizable structures but insufficient for diagnosis; point 3 indicated minimal criteria met for diagnosis, recognizable structures but with some technical or other flaws; point 4 indicated minimal criteria met for diagnosis, all structures imaged well and diagnosis easily supported; point 5 indicated minimal criteria met for diagnosis, all structures imaged with excellent image quality and diagnosis completely supported [18]. The scores from the two instructors were averaged.

\section{Outcomes}

The primary outcome included the diagnostic accuracy of the FASK protocol. The accuracy, sensitivity, specificity, positive predictive value (PPV), and negative predictive value (NPV) of the protocol were calculated that discharge/admission diagnosis was as the standard. The secondary outcomes included ED LOS and the rate of ED discharge using the FASK protocol.

\section{Statistical analysis}

All data were analyzed by SAS software (SAS 9.4, Cary, North Carolina, USA). Categorical data were expressed in counts and proportions, while continuous data were expressed in medians and interquartile ranges (IQRs). Categorical variables were compared using a Chi-square test. Continuous variables were examined using Wilcoxon's rank-sum test. A Kruskal-Wallis test was performed for comparisons for more than 2 groups.

Logistic regression was applied for possible factors for accurate sonographic diagnosis. Covariates in this model included age, sex, BMI, vital signs, registration time, the level of the sonographer, and the quality of US images. The level of the sonographer was categorized as junior physicians (PGY1-2) and senior physicians (PGY 3 or higher). Also, logistic regression was used for possible factors for the quality of the images. Covariates in this model included age, sex, BMI, vital signs, registration time, and the level of the sonographer. Odds ratio with $95 \%$ confidence intervals (Cls) for significant parameters were computed. A p-value of less than 0.05 was considered statistically significant.

\section{Results}




\section{Patient characteristics}

A total of 512 patients were included in the analysis (Fig. 1, Table 1). The median ages were 66 (IQR, 5677) years and 342 patients (67\%) were men. The median door to US time was 36 (IQR, 21-91.5) minutes. The median LOS was 165 (IQR, 96.5-1010) minutes. One patient experienced revisit within 72 hours for ascites tapping. No morbidity or death occurred. The median quality score of US images was $3($ IQR, 3$)$ and $78 \%(398 / 512)$ achieved acceptable quality (Likert scale $\geq 3)$.

Table 1

The characteristics of the included patients.

\begin{tabular}{|c|c|}
\hline Characteristics & $\begin{array}{l}\text { Total } \\
(n=512)\end{array}$ \\
\hline Age, years* & $66(56-77)$ \\
\hline Male, $n$ & $342(67 \%)$ \\
\hline Body mass index* & $23(20.4-25.5)$ \\
\hline \multicolumn{2}{|l|}{ Vital signs } \\
\hline Systolic blood pressure, $\mathrm{mm}-\mathrm{Hg}^{*}$ & $133(117-152)$ \\
\hline Diastolic blood pressure, $\mathrm{mm}-\mathrm{Hg}^{\star}$ & $75(66-84)$ \\
\hline Temperature, ${ }^{\circ} \mathrm{C} *$ & $37.1(36.8-37.4)$ \\
\hline Heart rate, per min* & $92(79-104)$ \\
\hline SpO2, \%* & $97(96-98)$ \\
\hline Door to US $^{\dagger}$ time, min* & $36(21-91.5)$ \\
\hline Imaging quality using a 5-point Likert scale* & $3(3)$ \\
\hline Patients receiving computed tomography, $\mathrm{n}$ & $81(16 \%)$ \\
\hline Length of stay, min* & $165(96.5-1010)$ \\
\hline $\mathrm{ED}^{\dagger}$ discharge, $\mathrm{n}$ & $435(85 \%)$ \\
\hline \multicolumn{2}{|c|}{ *Presented as median and interquartile ranges (IQRs). } \\
\hline${ }^{\dagger}$ US, ultrasonography; ED, emergency depart & \\
\hline
\end{tabular}

\section{Primary Outcome}

Comparing with the discharge/admission diagnosis, the accuracy of the FASK protocol was $99.0 \%$ (507/512). The sensitivity, specificity, PPV, and NPV were 99.4\% (95\% Cls, 98.2-99.9\%), 100\% (95\% Cls, 
100\%), 99.6\% (95\% Cls, 98.6-100\%), and 99\% (95\% Cls, 97.7-99.7\%), respectively. The disagreement between sonographic and discharge/admission diagnosis existed in only 5 patients: 2 patients had loculated ascites but PoCUS reported no presence of ascites; 2 had hydronephrosis but PoCUS reported no hydronephrosis; uterus was misinterpreted as an abdominal mass in one patient.

Logistic regression was applied for possible factors for accurate sonographic diagnosis. No significant parameters were identified to be associated with accuracy, whatever the level of the performer. Also, logistic regression was used for possible factors for image quality. Senior sonographers were associated with better image quality (Likert scale $\geq 3)(\mathrm{OR}, 1.72 ; 95 \% \mathrm{Cl}, 1.09-2.72)$. Others were unremarkable.

More than $70 \%$ of the patients had accurate diagnoses such as ascites or distended urinary bladder by using the FASK protocol (Fig. 1). Only $16 \%$ of the patients needed further advanced imaging.

There were 166 patients (32\%) diagnosed as having ascites, $212(41 \%)$ as having distended urinary bladder, and others had miscellaneous diagnoses (Fig. 1). The main causes of ascites were malignancy (49\%) and liver cirrhosis (44\%)(Table 2). One patient with ascites was diagnosed to have fresh endometrial cancer with peritoneal metastasis. Of patients with distended urinary bladder, $69 \%$ were related to benign prostate hypertrophy (Table 3). 
Table 2

The characteristics of the patients with ascites.

\begin{tabular}{|ll|}
\hline Variables & $\begin{array}{l}\text { Patients with ascites } \\
(\mathbf{n}=166)\end{array}$ \\
\hline Receiving CT*, $\mathrm{n}$ & $37(22 \%)$ \\
\hline Discharge, $\mathrm{n}$ & $130(78 \%)$ \\
\hline Length of stay, min ${ }^{\dagger}$ & $295(120-1578)$ \\
\hline Etiology, $\mathrm{n}$ & \\
\hline Malignancy & $81(49 \%)$ \\
\hline Hepatocellular carcinoma (HCC) & $25(15 \%)$ \\
\hline Colon cancer & $9(5 \%)$ \\
\hline Breast cancer & $9(5 \%)$ \\
\hline Ovarian cancer & $8(5 \%)$ \\
\hline Gastric cancer & $4(2 \%)$ \\
\hline Pancreatic cancer & $4(2 \%)$ \\
\hline Cholangiocarcinoma & $3(2 \%)$ \\
\hline Mesothelioma & $3(2 \%)$ \\
\hline Lung cancer & $3(2 \%)$ \\
\hline Others & $13(8 \%)$ \\
\hline Liver cirrhosis & $73(44 \%)$ \\
\hline Renal diseases & $5(3 \%)$ \\
\hline Ovarian hyperstimulation syndrome (OHSS) & $3(2 \%)$ \\
\hline Tuberculous peritonitis & $2(1 \%)$ \\
\hline Systemic lupus erythematosus & $2(1 \%)$ \\
\hline${ }^{*}$ CT, computed tomography. & \\
\hline${ }^{\dagger}$ presented with median (IQR). & \\
\hline
\end{tabular}


Table 3

The characteristics of the patients with distended urinary bladder.

\begin{tabular}{|c|c|}
\hline Variables & $\begin{array}{l}\text { Patients with distended } \\
\text { urinary bladder } \\
(n=212)\end{array}$ \\
\hline Receiving $\mathrm{CT}^{*}, \mathrm{n}$ & $6(3 \%)$ \\
\hline Discharge, $n$ & 201 (95\%) \\
\hline Length of stay, $\min ^{+}$ & $124(77.5-300.5)$ \\
\hline \multicolumn{2}{|l|}{ Etiology, $n$} \\
\hline Benign prostate hypertrophy & $146(69 \%)$ \\
\hline Malignancy & $20(9 \%)$ \\
\hline Prostate cancer & $11(5 \%)$ \\
\hline Bladder cancer & $4(2 \%)$ \\
\hline Others ${ }^{\ddagger}$ & $6(3 \%)$ \\
\hline Urinary tract infection & $11(5 \%)$ \\
\hline Miscellaneous§ & $34(16 \%)$ \\
\hline \multicolumn{2}{|l|}{${ }^{\star} \mathrm{CT}$, computed tomography. } \\
\hline${ }^{\dagger}$ presented with median (IQR). & \\
\hline
\end{tabular}

Sincluded drug side-effects (1), multiple sclerosis (1), neurogenic bladder (1),compression fractures (3), Foley dysfunction (3), and undetermined (25).

\section{Secondary Outcome}

397 patients (78\%) were discharged after receiving the FASK protocol and proper management. The median LOS was 126 minutes (IQR, 84-236 minutes). The remainder received further CT or management (Table 4). PoCUS was performed approximately 3 hours earlier than CT (median, 179 minutes; IQR, 90468 minutes; $\mathrm{p}<0.0001)$. 
Table 4

The comparisons of patient-centered outcomes between the patients with immediate discharge and those receiving further evaluation after the point-of-care ultrasound screening.

\begin{tabular}{|c|c|c|c|c|}
\hline & \multirow{2}{*}{$\begin{array}{l}\text { Discharge } \\
(n=397)\end{array}$} & \multicolumn{3}{|c|}{ Further evaluation } \\
\hline & & $\begin{array}{l}\text { CT } \\
(n=81)\end{array}$ & $\begin{array}{l}\text { Treatment } \\
(n=34)\end{array}$ & $p_{\text {Value }}$ \\
\hline $\begin{array}{l}\text { Door to } \mathrm{US}^{*^{\dagger}} \\
(\mathrm{min})\end{array}$ & $\begin{array}{l}33(19- \\
70)\end{array}$ & $59(26-226)$ & $\begin{array}{l}119(25- \\
330)\end{array}$ & $<.0001$ \\
\hline $\begin{array}{l}\text { Door to } \mathrm{CT}^{{ }^{+}} \\
(\mathrm{min})\end{array}$ & - & $\begin{array}{l}284(188- \\
685)\end{array}$ & - & \\
\hline $\begin{array}{l}\text { Length of } \\
\text { stay }^{\dagger}(\mathrm{min})\end{array}$ & $\begin{array}{l}126(84- \\
228)\end{array}$ & $\begin{array}{l}2335 \\
(1012- \\
4464)\end{array}$ & $\begin{array}{l}2484(1276- \\
4459)\end{array}$ & $\begin{array}{l}<.0001 \\
0.00\end{array}$ \\
\hline Discharge, $n$ & $\begin{array}{l}397 \\
(100 \%)\end{array}$ & $38(47 \%)$ & 0 & $<.0001$ \\
\hline \multicolumn{5}{|c|}{ *US, ultrasonography; CT, computed tomography. } \\
\hline
\end{tabular}

\section{Discussion}

There are diverse causes that contribute to abdominal distention and increase diagnostic complexity. This study showed a simple protocol, FASK, had efficacy and timeliness for the ED patients with abdominal distention. It could be an effective screening tool for the timely detection of ascites and distended urinary bladder, followed by proper management. The diagnostic accuracy of the protocol was $99 \%$ with high sensitivity and specificity, whatever the level of the performer. With the early intervention of the FASK protocol, the majority of patients could be discharged soon after proper management without further advanced imaging. It suggested that the introduction of PoCUS in patients with abdominal distention using a designated protocol could lessen LOS, facilitate the management process, and preserve safety. To our best of knowledge, this is the first study regarding the application of the PoCUS in non-critical patients with abdominal distention.

PoCUS is frequently used for critical patients, however, it has a positive impact on non-critical patients [5] This study also demonstrated the introduction of a simple US protocol with an easily accessible US machine could make incorporating PoCUS into the daily practice easy. In addition to being an extension of physical examination, PoCUS could play an important role in diagnosis and management in ED settings. Safety and timeliness, two domains of quality of care [19], would be preserved by using PoCUS.

Although US would be operator-dependent [20], no association could be identified between diagnostic accuracy and the level of the performer. Senior sonographers could achieve US images with better quality 
in this study, It could be explained that this protocol included two fundamental, simple US scanning techniques in US training. Also, junior sonographers performed the FASK protocol under the supervision of the instructors. The gap between the green hands and the experienced sonographers could be compensated. Therefore, the high accuracy, whatever the level of the sonographers could be explained.

Ascites can be easily identified by physical examination if the volume is greater than $1500 \mathrm{~mL}$ [21]. However, small amounts of fluid are not easily detected, or an increased adipose tissue may confound the diagnosis [22]. It would be safer with US-guided paracentesis [22, 23]. By contrast, paracentesis would be withheld if small amounts of ascites were detected. Also, urinary retention, often misdiagnosed as bowel obstruction or an abdominal tumor, is easily diagnosed by US [24]. In this study, more than $70 \%$ of the patients had ascites or a distended urinary bladder by using US, being the causes for their abdominal distention. Only $16 \%$ of the patients needed advanced imaging, such as CT for further evaluation.

The FAST examination is a commonly performed PoCUS examination to evaluate trauma patients in the ED. Besides detecting free fluid in the peritoneal cavity, indicating internal bleeding following trauma [25], the sonographer should be able to identify liver, kidney, spleen, and urinary bladder. The FAST examination can also provide valuable information in managing non-trauma patients and altering these patients' clinical course [26]. In this study, the FASK protocol included combinations of the FAST examination and the renal US. The simple protocol could be an effective, timely screening tool for patients with abdominal distention.

There was a certain percentage of the patients receiving CT following PoCUS. These patients experienced longer LOS and a lower discharge rate. The results showed that a certain percentage of patients needed further investigation besides PoCUS. It might be explained by the spirit of the PoCUS. PoCUS is the focused sonographic examination performed by the treating provider to inform clinical decision-making [27]. It is used to answer simple, binary (yes/no) questions [28]. Differences exist between PoCUS and comprehensive sonographic examination performing by established specialists [20], as well as CT. Also, these patients had longer door-to-US time and LOS, compared with those receiving US only. Possibly these patients were complicated to need more time for history taking, physical examination, and early management. Moreover, these patients had a lower rate of ED discharge and a higher probability of hospital admission.

There were several limitations. First, this was a retrospective, observational study with a non-random sample. Selection bias existed that the results were obtained from the formal US documentations. Some sonographic examinations without documentation were not included in the analysis. Besides, because PoCUS had become commonly used in EDs, ethical concerns raised about conducting a randomized controlled trial for PoCUS. Difficulties exist in estimating LOS without the introduction of US. Second, this study was conducted in a single center with an active US training program. The distribution of the causes of abdominal distention would vary from hospitals to hospitals. The external validity of the study results needs further verification. Third, the imaging quality was scored based on the still images in the US report. The still images could represent the quality of the sonographic examination partially. Dynamic evaluation 
of structures or position change of the patient may add significant information [29]. However, due to volume limitations, the US reporting system in our hospital could upload the still images. Fourth, the discharge/admission diagnosis having ascites or distended urinary bladder was used as a standard for accuracy. However, the underlying etiology for ascites or distended urinary bladder was not the main aim of the study. This study focused on the simple PoCUS protocol that would help in the quality of care and the management process. Last, this protocol could not detect loculated ascites outside the Morison's pouch, splenorenal recess, and the pelvis, as in 2 patients in this study.

\section{Conclusions}

A simple FASK protocol could be an effective screening tool for non-critical patients with abdominal distention. Being an adjunct of physical examination and management, PoCUS exhibited characteristics of efficacy, timeliness, and safety.

\section{List Of Abbreviations}

Ultrasound (US);

Point-of-care US (POCUS);

Length of stay (LOS);

Emergency department (ED);

Focused assessment with sonography in trauma (FAST);

Post-graduate year (PGY);

Body mass index (BMI);

Computed tomography (CT);

Interquartile ranges (IQRs);

Positive predictive value (PPV);

Negative predictive value (NPV);

Confidence intervals (Cls).

\section{Declarations}

Conflicts of interest: WC, JY, PH, DA, AF, YC, YT, CK, WT, CH, HP report no conflict of interest 
Ethics approval and consent to participate: The study was approved by the institutional review board of the National Taiwan University Hospital (201907173RIND) with a waiver of informed consent and registered at ClinicalTrials.gov (NCT04149041).

Consent for publication: The study was approved by the institutional review board of the National Taiwan University Hospital (201907173RIND) with a waiver of informed consent.

Availability of data and material: All data generated or analyzed during this study are included in this published article.

Competing interests: The authors declare that they have no competing interests.

Funding: None.

Authors' contributions: WC and HP conceived the study and designed the trial. WT, $\mathrm{CH}$, and HP supervised the conduct of the trial and data collection. WC, JY, PH, DA, AF, YC, YT, and CK undertook data collection and managed the data, including quality control. WC provided statistical advice on study design and analyzed the data; WC and HP chaired the data oversight committee. WC drafted the manuscript, and all authors contributed substantially to its revision. HP took responsibility for the paper as a whole.

Acknowledgments: None.

\section{References}

1. Malagelada JR, Accarino A, Azpiroz F. Bloating and Abdominal Distension: Old Misconceptions and Current Knowledge. Am J Gastroenterol. 2017;112(8):1221-31.

2. Jauhar S. The demise of the physical exam. N Engl J Med. 2006;354(6):548-51.

3. Bhagra A, Tierney DM, Sekiguchi H, Soni NJ. Point-of-Care Ultrasonography for Primary Care Physicians and General Internists. Mayo Clin Proc. 2016;91(12):1811-27.

4. Nielsen MB, Cantisani V, Sidhu PS, Badea R, Batko T, Carlsen J, et al. The Use of Handheld Ultrasound Devices - An EFSUMB Position Paper. Ultraschall Med. 2019;40(1):e1.

5. Weile J, Frederiksen CA, Laursen CB, Graumann O, Sloth E, Kirkegaard H. Point-of-care ultrasound induced changes in management of unselected patients in the emergency department - a prospective single-blinded observational trial Scand. J Trauma Resusc Emerg Med. 2020;28(1):47.

6. Sekiguchi $\mathrm{H}$. Tools of the Trade: Point-of-Care Ultrasonography as a Stethoscope. Semin Respir Crit Care Med. 2016;37(1):68-87.

7. Gillman LM, Kirkpatrick AW. Portable bedside ultrasound: the visual stethoscope of the 21 st century. Scand J Trauma Resusc Emerg Med. 2012;20:18.

8. ACEP. Ultrasound Guidelines. Emergency, Point-of-Care and Clinical Ultrasound Guidelines in Medicine. Ann Emerg Med. 2017;69(5):e27-54. 
9. Perera P, Mailhot T, Riley D, Mandavia D. The RUSH exam: Rapid Ultrasound in SHock in the evaluation of the critically III. Emerg Med Clin North Am. 2010;28(1):29-56.

10. Lichtenstein DA. BLUE-protocol and FALLS-protocol: two applications of lung ultrasound in the critically ill. Chest. 2015;147(6):1659-70.

11. Breitkreutz R, Price S, Steiger HV, Seeger FH, Ilper H, Ackermann H, et al. Focused echocardiographic evaluation in life support and peri-resuscitation of emergency patients: a prospective trial. Resuscitation. 2010;81(11):1527-33.

12. Lien WC, Hsu SH, Chong KM, Sim SS, Wu MC, Chang WT, et al. US-CAB protocol for ultrasonographic evaluation during cardiopulmonary resuscitation: validation and potential impact. Resuscitation. 2018;127:125-31.

13. Weile J, Laursen CB, Frederiksen CA, Graumann O, Sloth E, Kirkegaard H. Point-of-care ultrasound findings in unselected patients in an emergency department -results from a prospective observational trial. BMC Emerg Med. 2018;18(1):60.

14. Lynn AQ, Toce MS, Neal JT. Neonate with abdominal distention. Ann Emerg Med. 2019;73(4):e41-e2.

15. Melniker LA, Leibner E, McKenney MG, Lopez P, Briggs WM, Mancuso CA. Randomized controlled clinical trial of point-of-care, limited ultrasonography for trauma in the emergency department: the first sonography outcomes assessment program trial. Ann Emerg Med. 2006;48(3):227-35.

16. Ireton RC, Krieger JN, Cardenas DD, Williams-Burden B, Kelly E, Souci T, et al. Bladder volume determination using a dedicated, portable ultrasound scanner. J Urol. 1990;143(5):909-11.

17. Chan H. Noninvasive bladder volume measurement. J Neurosci Nurs. 1993;25(5):309-12.

18. ACEP. Emergency ultrasound standard reporting guidelines. 2018.

19. Bernstein SL, Aronsky D, Duseja R, Epstein S, Handel D, Hwang U, et al. The effect of emergency department crowding on clinically oriented outcomes. Acad Emerg Med. 2009;16(1):1-10.

20. Weile J, Brix J, Moellekaer AB. Is Point-Of-Care Ultrasound Disruptive Innovation? Formulating Why POCUS Is Different From Conventional Comprehensive Ultrasound Crit. Ultrasound J. 2018;10(1):25.

21. Sakai H, Sheer TA, Mendler MH, Runyon BA. Choosing the Location for Non-Image Guided. Abdominal Paracentesis Liver Int. 2005;25(5):984-6.

22. Keil-Ríos D, Terrazas-Solís H, González-Garay A, Sánchez-Ávila JF, García-Juárez I. Pocket ultrasound device as a complement to physical examination for ascites evaluation and guided paracentesis. Intern Emerg Med. 2016;11(3):461-6.

23. Nazeer SR, Dewbre H, Miller AH. Ultrasound-assisted paracentesis performed by emergency physicians vs the traditional technique. Am J Emerg Med. 2005;23(3):363-7.

24. Hata J. Point-of-Care Abdominal Ultrasound. Rinsho Byori. 2015;63(6):717-24.

25. Nordenholz KE, Rubin MA, Gularte GG, Liang HK. Ultrasound in the evaluation and management of blunt abdominal trauma. Ann Emerg Med. 1997;29(3):357-66.

26. Javedani P, Metzger G, Oulton J, Adhikar S. Use of Focused Assessment with Sonography in Trauma Examination Skills in the Evaluation of Non-trauma Patients. Cureus. 2018;10(1):e2076. 
27. Gleeson T, Blehar D. Point-of-Care Ultrasound in Trauma Semin Ultrasound. CT MR. 2018;39(4):37483.

28. Atkinson P, Bowra J, Lambert M, Lamprecht H, Noble V, Jarman B. International Federation for Emergency Medicine Point of Care Ultrasound Curriculum CJEM. 2015;17(2):161-70.

29. Jacobson JA, Khoury V, Brandon CJ. Ultrasound of the Groin: Techniques, Pathology, and Pitfalls. AJR Am J Roentgenol. 2015;205(3):513-23.

\section{Figures}

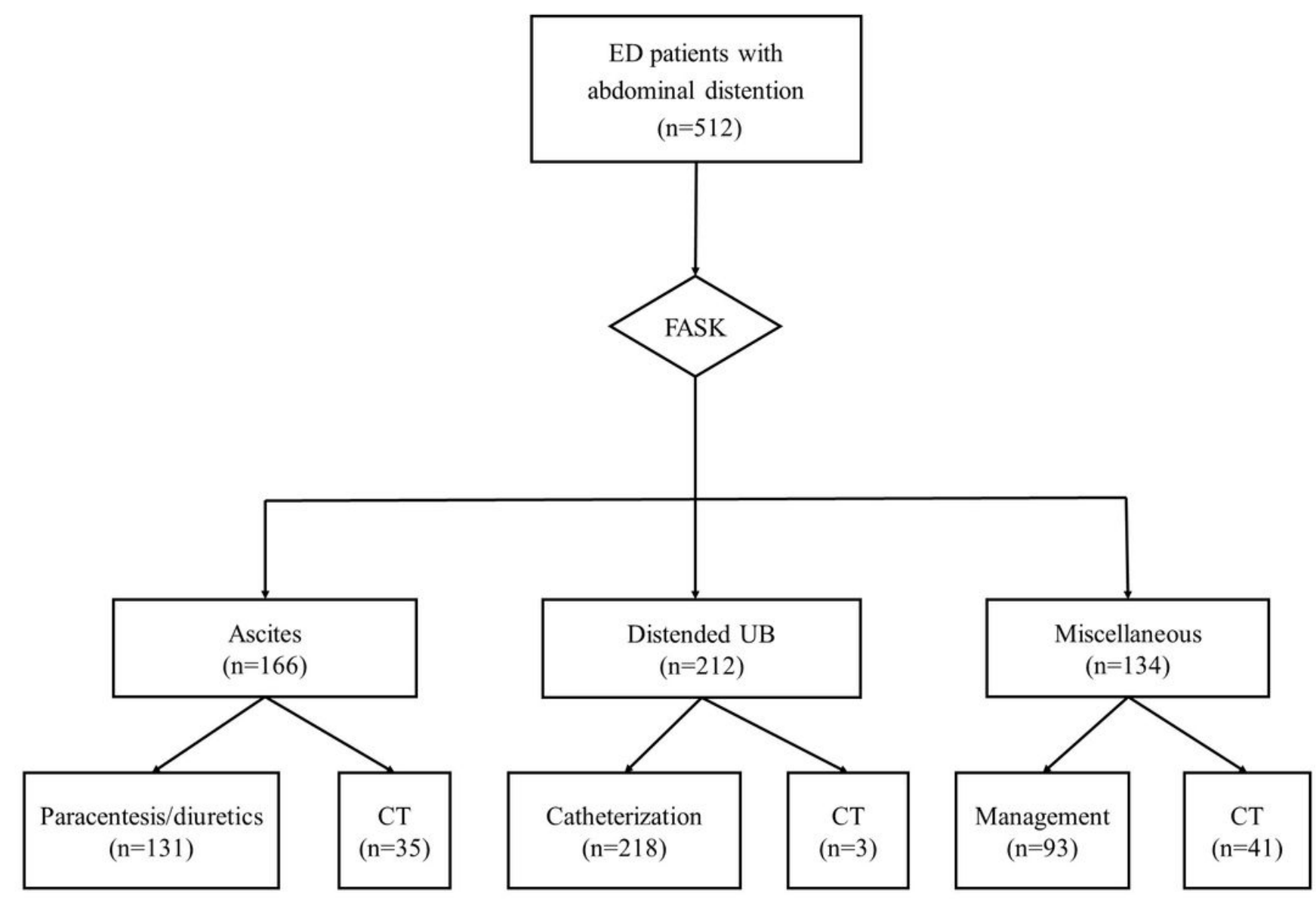

\section{Figure 1}

The study diagram.

\section{Supplementary Files}

This is a list of supplementary files associated with this preprint. Click to download. 
- STROBEchecklistcrosssectional0731.docx

Page 16/16 